\title{
Clinical response of dynamic splint using functional scales for the extension contracture of the metacarpophalangeal joint
}

\author{
Jun Nakayama ${ }^{1}$, Mituru Horiki², Kakuro Denno², Kazunori Ogawa ${ }^{3}$, \\ Hisao Oka ${ }^{4}$, Kazuhisa Domen ${ }^{5}$ \\ ${ }^{1}$ Department of Rehabilitation, Kansai Rosai Hospital \\ ${ }^{2}$ Department of Orthopaedics Surgery, Kansai Rosai Hospital \\ ${ }^{3}$ Daiya Gum Industry.CO \\ ${ }^{4}$ Graduate School of Health Sciencse, Okayama university \\ ${ }^{5}$ Department of Physical Medicine and Rehabilitation, Hyogo College of Medicine
}

\begin{abstract}
Collateral ligament shortening causes extension contractures of the metacarpophalangeal (MCP) joint, and dynamic flexion splinting (DFS) has been widely used to treat these contractures; however, there are various problems with these approaches. We developed a novel, pneumatic-type dynamic traction and flexion (DTF) splint to solve these problems. Twenty-one patients were treated with either the DTF or DFS for 8 weeks. Every 2 weeks, the average MCP joint flexion angle, total active motion (TAM), grasp strength, and pain scores were assessed. The flexion angle, in the DTF, was significantly greater than that in the DFS, starting after 6 weeks of treatment $(p<0.05)$. Similarly, the TAM results were significantly better following DTF treatment. Compared to treatment with DFS, our results showed that DTF treatment promoted earlier recovery of joint flexion angle and whole finger function in patients.
\end{abstract}

Keywords: extension contracture, dynamic splint, MCP joint

(Asian J Occup Ther 12: 85-91, 2016)

\section{Background}

Because finger function is important for holding and pinching objects, the metacarpophalangeal (MCP) joint is critical for the proper functioning of the hand. Contracture of these joints, often resulting from trauma and burns $[1,2]$, cause significant disorders in the activities of daily living (ADL), including eating, excretory functions, grooming and bathing. Thus, treatment of this type of contracture is extremely important for regaining normal hand function. Contractures of the MCP joints are common clinical conditions [3] that may be caused by shortening of the collateral ligament. Since the MCP joint is associated with a collateral ligament, unlike other joints, regaining flexibility of the collateral liga-

Received: 20 January 2015, Accepted: 10 December 2015

Corresponding to: Jun Nakayama, Department of Rehabilitation, Kansai Rosai Hospital, 69-1, Inabaso, Amagasakishi, Hyogo, 6608511, Japan

e-mail: nakayama-kanrou.hp@live.jp

C2016 Japanese Association of Occupational Therapists ment is necessary to improve the joint's range of motion. However, treatment of severe contractures is often very difficult.

Appropriate treatment of this type of contracture is extremely important. The therapy used to treat the joint contracture should be both integrative and problem-focused. Therefore, the therapist should avoid worsening the joint problem through inappropriate rehabilitation, such as long-term fixation and malpositioning of the joint after a fracture. Pressure therapy, active mobilization, and passive mobilization, using remedial orthotic positioning, should be started as soon as a problem is diagnosed. Continuous stretching is an effective treatment for these contractures [4], and the treatment efficacy is improved through the use of a dynamic orthosis [5-7], which is a well-accepted modality used to regain joint motion in an injured hand [8]. The clinical success of dynamic orthotic positioning is better and results in less joint stiffness if the interval between injury and treatment is kept short [9].

The dynamic flexion splint (DFS-splint) has been widely used for the treatment of MCP joint extension 
contractures $[10,11]$. This conventional type of flexion splint can, however, negatively impact the articular surface of the joint, leading to pain when the finger is flexed for an extended period [3]. As a result, range of motion improvements are difficult to obtain [11]. Therefore, we devised a new dynamic traction splint using curved rubber, also known as pneumatic artificial muscle (PAM), as part of a novel dynamic splint. PAM is used for rehabilitations involving remedial neuromuscular and gate exercises [12-14], including for providing power assistance to paralyzed muscles [15]. Also, PAM is used in conjunction with various joints as an adjunct for weak or paralyzed muscles, according to preliminary research [16]. However, the use of PAM splints has not been previously reported for improving joint contracture.

We devised a novel pneumatic-type dynamic traction and flexion (DTF-splint) splint [17] that pulls the articular surface and corrects flexural direction by combining PAM with a dynamic splint. This study investigated the flexion angle, total active motion (TAM) score, grasp strength, and pain (using a Visual Analog Scale [VAS] score) associated with wearing the splint by patients with MCP joint extension contractures; patients were randomly assigned to use either the DTF or conventional DFS-splint. Additionally, we examined the patients' clinical responses to the use of both splints

\section{Methods}

\section{Study design and ethics}

The trial protocol was approved by our hospital's ethics committee on human research. Written informed consent was obtained from all patients who agreed to participate in the research protocol was obtained prior to their enrollment in the study.

\section{Patients}

We evaluated 21 patients (52 fingers) with extension contractures of the MCP joints, less than six months after the contractures developed. A total of 13 men (29 fingers) and 8 women (23 fingers) were treated with either DFS or DTF-splints. The average age of the patients treated with DFS-splints was 42-years-old (range, 28-59-years old), and 49-years-old (range, 33-62-yearsold) for those treated with DTF-splints. The contractures developed as a result of proximal phalanges fractures (5 patients, 6 fingers), metacarpal bone fractures (2 patients, 2 fingers), proximal phalanges and metacarpal bone fractures ( 4 patients, 8 fingers), extensor tendon injuries (3 patients, 8 fingers), distal radius fractures (4 patients, 16 fingers) and complex regional pain syndrome type II ( 3 patients 12 fingers). The subject fingers included 15 index, 7 middle fingers, 8 ring fingers and
22 little fingers. The affected fingers were treated with osteosynthesis (15 patients, 32 fingers) or conservatively (6 patients, 20 fingers) (Table 1).

The patients were directed to wear the assigned splint for their range of motion exercises. In all cases, the patients started wearing the splints when their elastic traction was 200 gf [4, 18-20]. The authors assisted patients to put it on every day for eight weeks. The splint had to be worn by the patients thrice in a day, in the morning, noon and night, for 30 minutes each. An occupational therapist checked each patient's traction power and appropriate wearing of the splint every 2 weeks.

\section{Measurements}

The subjects were randomly assigned to use either the GDS or DTF-splints. I carried out blind in Block Randomization. We evaluated the progress of the TAM scores (Total flexion angles - extension lag) and MCP joint flexion angles, every two weeks, over an eightweek period. TAM is used as one of the evaluation methods of the finger dysfunction. Further, we compared

Table 1. Characteristics of the patients with extension contractures of the MCP joints

\begin{tabular}{|c|c|c|c|c|}
\hline & \multicolumn{2}{|c|}{ DTF } & \multicolumn{2}{|c|}{ DFS } \\
\hline & $\begin{array}{l}\text { Patients } \\
\text { (n) }\end{array}$ & $\begin{array}{l}\text { Fingers } \\
\text { (n) }\end{array}$ & $\begin{array}{l}\text { Patients } \\
\text { (n) }\end{array}$ & $\begin{array}{l}\text { Fingers } \\
\text { (n) }\end{array}$ \\
\hline \multicolumn{5}{|l|}{ Sex } \\
\hline Male & 5 & 11 & 8 & 18 \\
\hline Female & 4 & 14 & 4 & 9 \\
\hline total & 9 & 25 & 12 & 27 \\
\hline \multicolumn{5}{|l|}{ Age (yrs) } \\
\hline Male & 46.4 & NA & 43.8 & NA \\
\hline Female & 51.2 & NA & 46.3 & NA \\
\hline average & $\begin{array}{c}49 \\
(33-62)\end{array}$ & NA & $\begin{array}{c}42 \\
(28-59)\end{array}$ & NA \\
\hline \multicolumn{5}{|l|}{ Disease } \\
\hline $\begin{array}{l}\text { Proximal phalanges and } \\
\text { Metacarpal bone fracture }\end{array}$ & 2 & 5 & 2 & 3 \\
\hline Extensor injure & 1 & 4 & 2 & 4 \\
\hline Distal radius fracture & 2 & 8 & 2 & 8 \\
\hline CRPS type1 & 1 & 4 & 2 & 8 \\
\hline total & 9 & 25 & 12 & 27 \\
\hline \multicolumn{5}{|l|}{ Treatment type } \\
\hline Osteosynthesis & 6 & 13 & 9 & 19 \\
\hline Conservative & 3 & 12 & 3 & 8 \\
\hline total & 9 & 25 & 12 & 27 \\
\hline \multicolumn{5}{|l|}{ Subject } \\
\hline Index & NA & 7 & NA & 8 \\
\hline Middle & NA & 3 & NA & 4 \\
\hline Ring & NA & 4 & NA & 4 \\
\hline Little & NA & 11 & NA & 11 \\
\hline total & NA & 25 & NA & 27 \\
\hline
\end{tabular}


the two groups for their ability to grasp and their experience with sharp pain (VAS score) when they started treatment with their assigned splint, and after 8 weeks of progress. The evaluator was an occupational therapist blinded to the purpose of the research. In addition, we carried out three times of evaluations. We calculated three times of means.

\section{Structure and characteristic of the DFS and DTF-splints}

DFS-splint. The DFS-splint comprised a thermoplastic support ( $3 \mathrm{~mm}$, no hole; OG Giken, Okayama, Japan), a Velcro strap and an elastic band (Figure 1A). The cock-up splint fixed the location of the wrist joint and metacarpal bones; the subject finger was positioned inside a glove that allowed the tip of the finger to be directly pulled, using the power of the elastic band, to flex the finger at the MCP joint.

DTF-splint. The DTF-splint comprised a wrist joint dorsiflexion instrument, a curved PAM and wrist jointarthrodesis belt (Daiya Industrial, Okayama, Japan), a miniature gas cylinder (74 g, Nippon Tansan Gas, Tokyo, Japan), a Velcro strap and an elastic band (Figure 1B). A profile view of the splint (Figure 2) shows the characteristics of this splint that pulls the articular surface and corrects the flexural direction by applying a PAM to the dynamic splint; the splint permits the treatment of two or more fingers, simultaneously. The PAM used in this study is made of curved rubber (Figure $3 \mathrm{~A}$ and $3 \mathrm{~B}$ ) and comprises a rubber tube covered with a bellows sleeve that only extends or contracts axially. By inhibiting the extension of one side, with the fiber reinforcement, the bending motion in the desired direction occurs by supplying compressed air to the rubber tube. As shown in Figure 3A, the curved tube is covered

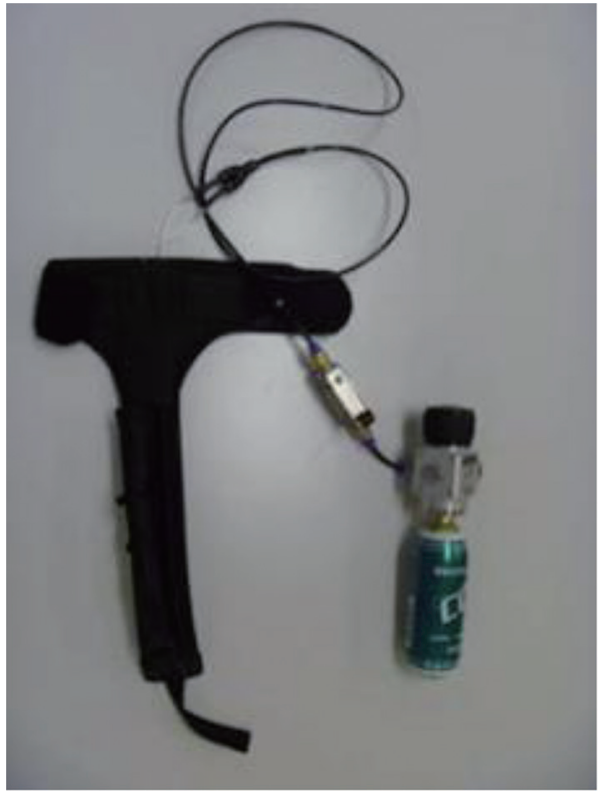

Fig. 2. The PAM used in this study is the curved type pneumatic rubber artificial muscle.

by an inner, non-contracting cloth and an outer, rubber cloth. The curved, rubber PAM bends when the rubber tube expands as result in the difference of the lengths of the rubber and cloth (outer and inner) covers. The bending motion was used to flex the joint, and the extension movement was used for joint traction (Figure 3B). The subject finger was wrapped to avoid any possibility of local ischemia.

\section{Statistical methods}

Statistical analyses were performed using the Kruskal-Wallis and Bonferroni methods for making within-group comparisons, and the Mann-Whitney

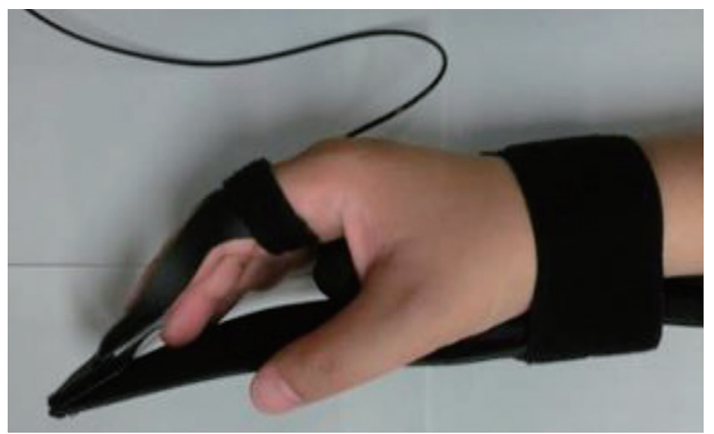

(b) Pneumatic-Type Dynamic Traction and flexion splint (DTF splint)

Fig. 1. The dynamic splint used in this study.

A) Dynamic flexion splint; Couk-up splint fixed at the wrist joint and metacarpal bones. The flexion-type equip gloves on the subject's finger, pulls the tip of the finger directly in and causes flexion at the MP joint due to the rubber.

B) Pneumatic-Type Dynamic Traction and flexion splint: A characteristic motion of the artificial muscle is made possible such that extension and bending of the fingers takes place. The bending motion was used for flexion of the joint, and the movement of the extension was used for traction of the joint. 

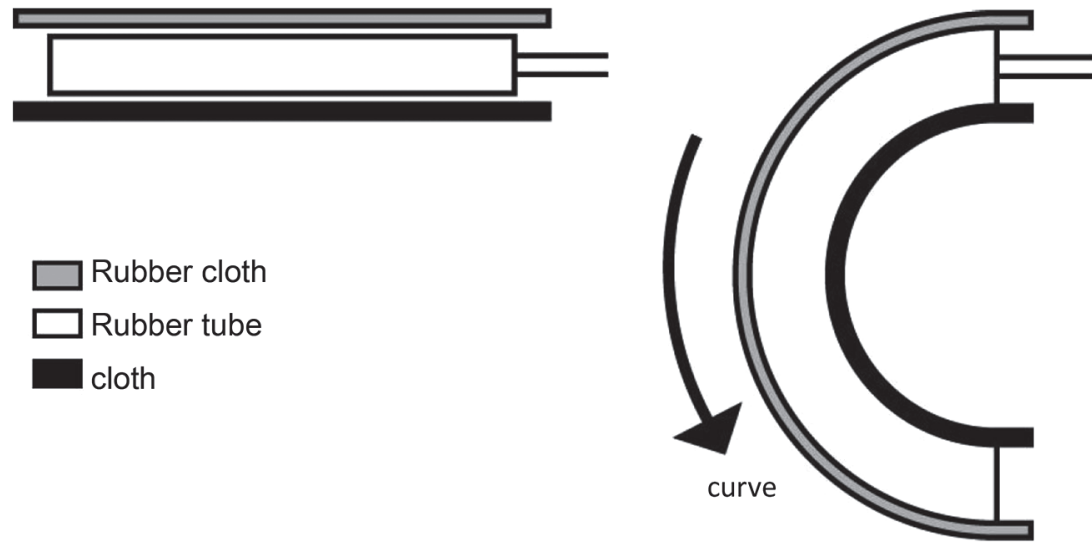

(a)No pressurized

(b)Pressurized

Fig. 3. Structure of the curved type pneumatic rubber artificial muscle.

A) Non pressurized rubber tube.

B) Pressurized curved rubber tube.

U-test was used to perform between-group comparisons. The significance of the differences between mean values was calculated. All statistical analyses were performed using the SPSS Base 11.0J statistics software (SPSS Japan, Tokyo, Japan); P-values $<0.05$ were considered statistically significant.

\section{Results}

Table 2 shows the details of the effects of wearing both splint types over the 8 -week treatment period.

\section{MCP joint flexion angle}

The pretreatment MCP joint flexion angles were $27.3 \pm 10.8^{\circ}$ and $23.3 \pm 8.9^{\circ}$ in the DFS and DTF-splint groups, respectively. In the DTF-splint group, the MCP joint flexion angles improved significantly more, over the 8-week treatment, than those in the DFS-splint group $(P<0.05$, Table 2). Furthermore, the range of motion improvement was significantly improved in the group treated with the DTF-splint for 8 weeks, compared to those treated with the DFS-splint $(P<0.01$, Table 2$)$.

\section{TAM scores}

The pretreatment TAM scores for the DFS- and DTF-splint groups were $175.3 \pm 19.8^{\circ}$ and $171.5 \pm 27.2^{\circ}$, respectively. Over the 8 -week treatment period, the DTF-splint group showed significantly improved flexion angles compared with the DFS-splint group $(P<0.05)$. Furthermore, the TAM improvement was significantly greater for those wearing the DTF-splint than for those wearing the DFS-splint $(P<0.01)$.
Table 2. Evaluation results in relation to the use of dynamic flexion splint (DFS) and pneumatic-type dynamic traction and flexion (DTF).

\begin{tabular}{lcccc}
\hline & Evaluation & DFS & DTF & P value \\
\hline Joint flexion $\left(^{\circ}\right)$ & pretreatment & $27.3 \pm 10.8$ & $23.3 \pm 8.9$ & 0.65 \\
& final & $60.1 \pm 9.8$ & $65.1 \pm 12.3$ & $* 0.04$ \\
& gain & 32.8 & 41.8 & ${ }^{\dagger} 0.01$ \\
\hline \multirow{2}{*}{ TAM } & pretreatment & $175.3 \pm 19.8$ & $171.5 \pm 27.2$ & 0.72 \\
& final & $207.9 \pm 10.9$ & $218.7 \pm 12.3$ & $* 0.02$ \\
& gain & 32.6 & 47.2 & 0.01 \\
\hline \multirow{2}{*}{ Grasp $(\mathrm{kg})$} & pretreatment & $5.8 \pm 6.1$ & $5.2 \pm 3.9$ & 0.89 \\
& final & $25.3 \pm 9.8$ & $22.6 \pm 10.7$ & 0.61 \\
& gain & 19.5 & 17.4 & 0.08 \\
\hline \multirow{2}{*}{ VAS-score } & pretreatment & $2.2 \pm 1.0$ & $2.5 \pm 0.5$ & 0.54 \\
& final & $1.2 \pm 0.6$ & $1.1 \pm 0.7$ & 0.43 \\
& gain & 1 & 1.4 & 0.71 \\
\hline
\end{tabular}

* P values $<0.05$ were considered statistically significant.

${ }^{\dagger}$ P values $<0.01$ were considered statistically significant.

\section{Grasp strength}

The mean grasp strength of the patients wearing the DTF-splint was not significantly different from that for the patients wearing the DFS-splint, at the end of the treatment period. Hence, the improvements in the two groups were similar.

\section{VAS-score}

Similarly, there were no significant differences in VAS pain scores between the groups wearing the DFS and DTF-splints.

The 2-week rates of improvement for the patients wearing both types of splints 


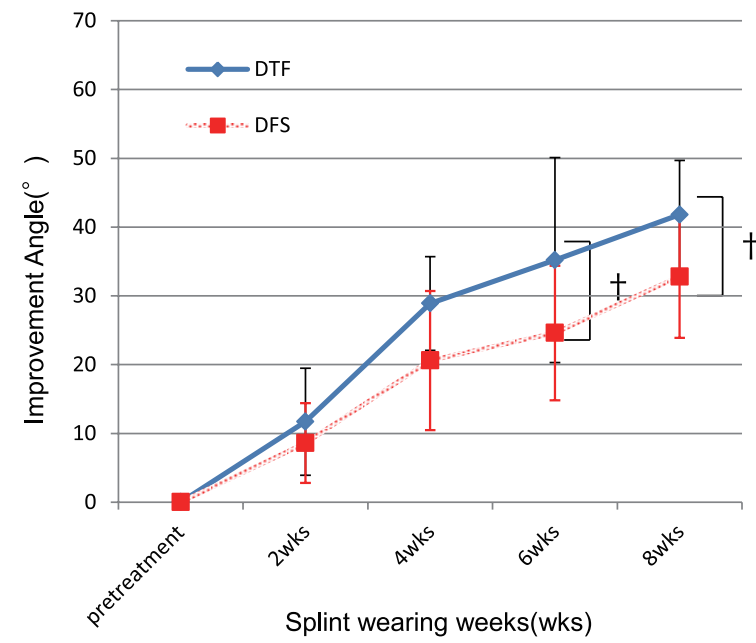

(a) MP joint flexion angle

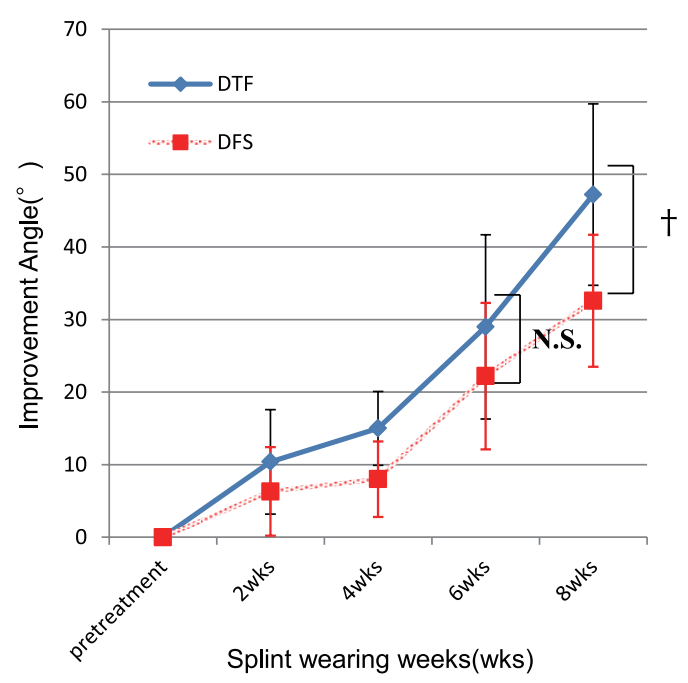

(b) Total Active Motion-score

Fig. 4. The 2-week improvement rates in patients wearing DFS and DTF splint.

We dissected 21 patients 52 fingers. (A) was the MP joint flexion angle, (B) was the total Active Motion-score. The significance level was set at ${ }^{*} p<0.05^{\dagger} p<0.01$.

MCP joint flexion angle improvement angle (Figure 4A)

As shown in Figure 4A, for the first 4 weeks, there was no significant difference between the DTF and DFS-splint groups. However, patients wearing the DTFsplint improved significantly faster by weeks 6 and 8 than did the patients in the DFS-splint group $(P<0.01)$.

\section{TAM score improvement angle (Figure 4B)}

The TAM scores, over the first 6 weeks of treatment, were not significantly different between the two groups However; the DTF-splint group improved significantly more during the last two weeks of treatment than did the DFS-splint group ( $P<0.01$, Figure 4B).

\section{Discussion}

Generally, extension contractures are the most common contractures of the MCP joint. Maintaining maximum finger range of motion during the early treatment of these contractures is important to achieve optimal outcomes. Contracture of the MCP joint is a common clinical condition [17], but treatment is difficult and requires more time when the contracture is fully formed. This is the reason that the contractures should be treated as early as possible. There are several possible causes of joint contractures, including adhesions of the extensor tendons over the dorsum of the hand or of the extensor hood mechanism over the MCP joint; thickening of the dorsal MCP joint capsule; contracture of the collateral ligament; insufficient skin coverage or scarring of the skin over the dorsum of the hand, as in a burn; or a bony block within the joint [21, 22]. Of these, the most common cause of extension contractures of the MCP joint is shortening of the collateral ligament [11].

The dynamic splint is a well-accepted modality used for restoring joint motion to an injured hand. However, in extension contractures that involve a bent MCP joint, such treatment may not only be ineffective, but may also injure the articular cartilage causing greater damage. Therefore, thermotherapy is an effective treatment adjunct, prior to the use of a joint traction method [11]. This study assessed whether the DTF-splint is an effective device for treating MCP joint extension contractures. We used both the novel DTF-splint and the conventional DFS-splint to treat patients with extension contractures of the MCP joint. The results indicated that the DTF-splint yielded early recovery of both joint flexion angle and finger function. However, the changes in grasp strength and VAS scores were not significantly different between the DTF and DFS-splint treatments. Specifically, the improvement angle in the range of motion of the joint flexion angle was significantly improved at both 6 and 8 weeks of treatment, compared to joints treated using a DFS-splint. As a result, the TAM scores in joints treated using the DTF-splint were also significantly improved by week 8 , compared to joints treated using the DFS-splint.

Generally, a PAM enables effective treatment to be delivered under conditions of relatively low pressure and reduced tension; PAM provided powered torque to the splint in the DTF-splint. Additionally, the utilization of PAM enables the use of a splint that is flexible, lightweight, easily maintained, and inexpensive. Recent studies have quantified the force-length, force-velocity, 
force-activation, and bandwidth properties of artificial pneumatic muscles in detail [23-26]. The low compliance and high of the PAM is well suited for mimicking natural gait movements $[24,25]$ and, in the current orthosis, for providing a power-assisted glove [18].

According to our preliminary research, the joint space in the most palmar part of the MCP joint was increased by approximately $1.7 \mathrm{~mm}$, using the DTF-splint compared to the joint, without orthotic treatment [18]. The DFS-splint works by adding tension to the joint, in the flexional direction, using elastic power to the fingertip. On the other hand, the DTF-splint allows extension and bending of the fingers. The bending motion was used to flex the joint, and extension was enabled using joint traction. Thus, the DTF-splint allows extension of the extensor tendon as well as the application of traction. The improved flexion angle of the whole finger, into the interphalangeal joint, was accomplished using the PAM along the full length of a finger. As a result, there was improvement in both the extension and flexion of the whole finger, including the interphalangeal joint.

The traction force of the DTF-splint extended the collateral ligaments of the MCP joints. This may indicate an improvement in the overall function of the finger as well as an improvement in the flexural angle, compared to fingers treated with a DFS-splint. However, as for the splints, a grip and a pain did not have a change. Furthermore, the effect in DTF-splint is not immediate, and it is actualized several weeks later. We regulated power of traction while being careful about sharp pain. Therefore, we think that stretch of tissue might take time. That is why there is not the DTF-splint immediate effect. However, it is the DTF-splint may be an effective tool for improving a patient's digital range of motion in comparison with DFS-splint several weeks later.

\section{Study limitations}

This study has a few limitations. First, each patient used the splint for only 8 weeks, rather than over a long period. Hence, we were unable to determine the period of treatment. Second, only patients who had been diagnosed with MCP joint contractures within 6 months of the start of the study were enrolled. Third, we did not evaluate the impact of the treatment on the activities of daily living. Finally, the study involved a limited number of patients. In future studies, we plan to increase number of patients and test the long-term effects of the splint and the usefulness of the splint for treating contractures diagnosed more than 6 months prior to the start of therapy.

\section{Conclusion}

Our results show that use of the novel DTF splint allows early improvements in the overall flexion of a finger and in the MCP joints flexural angle in patients with MCP joint extension contractures. Additionally, in this limited study, the DTF-splint appeared to be more effective than DFS-splints for treating patients with extension contractures of the MCP joint.

Acknowledgment: This study has received a grant from the Okayama Prefecture Industrial Promotion Foundation in 2011.

\section{References}

[1] Choi JS, Mun JH, Lee JY. Effects of Modified Dynamic Metacarpophalangeal Joint Flexion Orthoses after Hand Burn. Ann Rehabil Med. 2011; 35(6): 880-6.

[2] Yi N, Wang BS, Zhu XX. Application with a series of orthotic splints for recovery of hand function after burn. Chinese Journal of Burns. 2008; 24(3): 191-4.

[3] Ueba Y, Kurata H, Ono I. Basic biomechanics for orthotic therapy of the hand. Bulletin of the Japanese Society of Prosthetics and Orthotics. 1999; 15: 119-24.

[4] Shibata K, Ikuta M, Nomura T. Relation between traction force and cuff shape of splint on the peripheral blood flow of the digits. Japanese Occupational Therapy Research. 1986; 7: 485-6.

[5] Wong JMW. Management of stiff hand: an occupational therapy perspective. Hand Surg. 2002; 7(2): 261-9.

[6] Flowers KR, LaStayo P. Effect of total end range time on improving passive range of motion. J Hand Ther. 1994; 7(3): $150-7$.

[7] Judith A. Rehabilitation of the hand. St. Louis: The CV Mosby Company; 1978; 322-9.

[8] Colditz JC. Low profile dynamic splinting of the injured hand. Am J Occup Ther. 1983; 37(3): 182-8.

[9] Glasgow C, Tooth LR, Fleming J, et al. Dynamic splinting for the stiff hand after trauma: predictors of contracture resolution. J Hand Ther. 2011; 24(3): 195-206.

[10] Tajima T. Treatment of post-traumatic contracture of the hand. The Journal of Hand Surgery, British \& European. 1988; 13(2): 118-29.

[11] Nakayama J, Kurokawa K, Konishi A, et al. A trial of a revolving-type dynamic traction splint for extension contracture of the metacarpophalangeal joint. Japanese Occupational Therapy Research. 2008; 27: 168-73.

[12] Do Nascimento BG, Vimieiro CBS, Nagem DAP, et al. Hip orthosis powered by pneumatic artificial muscle: Voluntary activation in absence of myoelectrical signal. Artif Organs. 2008; 32(4): 317-22.

[13] Gordon KE, Sawicki GS, Ferris DP. Mechanical performance of artificial pneumatic muscles to power an anklefoot orthosis. J Biomech. 2006; 39(10): 1832-41.

[14] Park YL, Chen B-r, Pérez-Arancibia NO, et al. Design 
and control of a bio-inspired soft wearable robotic device for ankle-foot rehabilitation. Bioinspiration \& Biomimetics. 2014; 9(1): 016007.

[15] Sasaki D, Noritsugu T, Yamamoto H, et al. Development of power assist wear using pneumatic rubber artificial muscles. Journal of Robotics and Mechatronics. 2009; 21(5): 607.

[16] Nagai K, Nakanishi I, Hanafusa H. Assistance of selftransfer of patients using a power-assisting device. International Conference on Robotics and Automation. 2003.

[17] Nakayama J, Horiki M, Ogawa K, et al. Development of a new dynamic traction and flexion splint for MCP joint extension contractures. Tokyo: Keiogijyuku; 2014.

[18] Stewart KM. Review and comparison of current trends in the postoperative management of tendon repair. Hand Clin. 1991; 7(3): 447-60.

[19] Mildenberger LA, Amadio PC, An KN. Dynamic splinting: a systematic approach to the selection of elastic traction. Arch Phys Med Rehabil. 1986; 67(4): 241-4.

[20] Nuismer BA, Ekes AM, Holm MB. The use of low-load prolonged stretch devices in rehabilitation programs in the Pacific northwest. Am J Occup Ther. 1997; 51(7): 538-43.
[21] Curtis RM. Hand splinting, principles and methods. St. Louis: The C V Mosby Company; 1981.

[22] Jacobs MLA, Austin N, Austin NM. Splinting the hand and upper extremity: Principles and process: Lippincott Williams \& Wilkins; 2003.

[23] Davis S, Tsagarakis N, Canderle J, et al. Enhanced modelling and performance in braided pneumatic muscle actuators. The International Journal of Robotics Research. 2003; 22: 213-27.

[24] Klute GK, Czerniecki JM, Hannaford B. Artificial muscles: Actuators for biorobotic systems. The International Journal of Robotics Research. 2002; 21(4): 295-309.

[25] Klute GK, Hannaford B. Accounting for elastic energy storage in McKibben artificial muscle actuators. Journal of Dynamic Systems, Measurement, and Control. 2000; 122(2): 386-8.

[26] Reynolds DB, Repperger DW, Phillips CA, et al. Modeling the dynamic characteristics of pneumatic muscle. Ann Biomed Eng. 2003; 31(3): 310-7. 